\title{
Bronchoscopic Advances: On the Way to the Cells
}

\author{
Luc Thiberville Mathieu Salaün
}

Clinique Pneumologique, Rouen University Hospital, and QuantIF-LITIS EA 4108, Faculté de Médecine-Pharmacie, Rouen University, Rouen, France

\section{Key Words}

Bronchoscopy - Autofluorescence - Narrow band imaging • Optical coherence tomography $\cdot$ Fluorescence confocal microendoscopy $\cdot$ Microimaging, in vivo $\cdot$ Pulmonary alveoli

\begin{abstract}
In the past 15 years, new endoscopic methods have been developed in order to improve the detection of early bronchial cancers, with autofluorescence bronchoscopy being the leading technique. However, autofluorescence bronchoscopy is hampered by the low specificity of the fluorescence defect which ranges from 25 to $50 \%$, and its limitation to the proximal bronchial tree from which arise only half of the lung cancers that are currently diagnosed. To overcome these limitations, other techniques emerge including video/autofluorescence bronchoscopy, narrow band imaging, optical coherence tomography, and 'endomicroscopy' using confocal fluorescent laser microscopy. These emerging techniques provide new insight into bronchology, extending the field of exploration from the proximal bronchus down to the most distal part of the lungs, and from macroscopy to in vivo cellular imaging. In the near future, they may enable in vivo, minimally invasive, 'pathological grade' evaluation of abnormal bronchial or parenchymal lung tissue. Whereas promising pioneer work has recently been published, careful assessment is required before these methods find a place in the evaluation strategy of early lung cancer and other lung diseases.
\end{abstract}

Copyright $\odot 2010$ S. Karger AG, Basel

\section{KARGER}

Fax +4161306 1234

E-Mail karger@karger.ch

www.karger.com

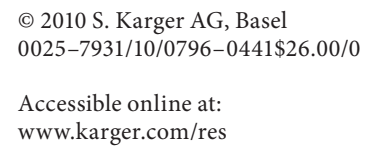

\section{Introduction}

Bronchoscopy is the essential tool for the diagnosis and management of centrally located lung cancer. Until recently, the early diagnosis of these epithelial changes relied primarily on gross inspection of the bronchial mucosae during an endoscopic procedure and pathology examination of biopsy samples derived from the macroscopy. Classic fiberoptic bronchoscopy, using white-light illumination, has repeatedly shown a low sensitivity for the detection of the early - presumably curable - lesions such as carcinoma in situ (CIS) [1].

In the past 15 years, methods have been developed in order to improve the detection of these early cancerization stages, with autofluorescence (AF) bronchoscopy (AFB) being the major breakthrough $[2,3]$. Following pioneer work from the British Columbia Cancer Center, the

Previous articles in this series: 1. Herth FJF, Gompelman D, Ernst A, Eberhardt R: Endoscopic lung volume reduction. Respiration 2010;79:5-13. 2. Lee P, Mathur PN, Colt HG: Advances in thoracoscopy: 100 years since Jacobaeus. Respiration 2010;79:177-186. 3. Eberhardt R, Kahn N, Herth FJF: 'Heat and destroy': bronchoscopic-guided therapy of peripheral lung lesions. Respiration 2010;79:265-273.

Funded by: Programme Hospitalier de Recherche Clinique 2007, French Ministry of Health; Canceropole Nord Ouest, Lille, France; Institut National du Cancer, (Grant INCa-Lilly No. 0612-3D1317-31), Boulogne Billancourt, France, and ADIR Association, Bois Guillaume, France.
Prof. L. Thiberville

Clinique Pneumologique, Hôpital Charles-Nicolle, Rouen University Hospital 1 rue de Germont

FR-76000 Rouen (France)

Tel. +3323288 82 47, Fax +332328882 40, E-Mail Luc.Thiberville@ univ-rouen.fr 
use of blue light/AFB associated with white-light/conventional bronchoscopy has been developed, consistently showing high sensitivity in detecting precancerous bronchial lesions, i.e. moderate dysplasia or higher grade lesions. AFB is based on the observation that premalignant and malignant bronchial mucosae fluoresce less than normal tissue, and thereby allow detection of lesions, i.e. CIS, that may have a normal appearance during conventional white-light bronchoscopy. However, the technique is hampered by the low specificity of the fluorescence defect which ranges from 25 to $50 \%$. This is due to the fact that AFB using fiberoptic bronchoscopes is unable to differentiate early precancerous lesions from inflammatory changes in the bronchial tree. Another difficulty of an early bronchoscopic detection approach is related to its limitation to the proximal bronchial tree, from which arise only half of the lung cancers that are currently diagnosed.

To overcome these limitations, other techniques have emerged over the past 5 years that extend the field of exploration of bronchoscopy to the distal lung and to the cellular level. These new bronchoscopic modalities take advantage of recent advances in computer technology and engineering, and may challenge or be advantageously associated with AFB in the early detection and staging of lung cancer.

These new tools include video/AFB, narrow band imaging (NBI), optical coherence tomography (OCT), and 'endomicroscopy' using confocal fluorescent laser microscopy or cytoscopy using very high magnification.

\section{Modern 'Macro' Endoscopic Approach: Video-Autofluorescence and Narrow Band Imaging}

\section{A New Look to Bronchial Fluorescence: \\ Video Autofluorescence Systems}

The initial and still most commonly used AFB systems are based on fiber-optic bronchoscopes associated with a CCD camera. As fiber-optic bronchoscopy tends to be replaced by video-endoscopy at most bronchoscopy facilities, AF diagnosis systems integrated into video-endoscopes have been developed. These systems include in their design new tools in order to improve the imaging specificity for precancerous lesions. Two video-AF systems are currently available. Both systems have been evaluated in a small number of patients and showed higher sensitivity for intraepithelial lesions than white light video-endoscopy alone.
The SAFE $3000^{\circledR}$ system (Pentax, Tokyo, Japan) uses both a xenon lamp for white light imaging and a monochromatic diode laser for fluorescence imaging. As both light sources are available at the same time, the system enables dual real-time imaging where both video white light and AFB images are displayed simultaneously [2]. Besides the convenience of such a system which allows more precise targeting of biopsies, one study showed that dual imaging using SAFE 3000 improves the specificity of the AF imaging [3].

In a recent study, Usuda et al. [4] have used the SAFE 3000 system immediately before diode laser illumination for photodynamic therapy using a second generation photosensitizer administered intraveneously, NPe6. They showed that the tumor margin could be accurately defined before photodynamic therapy, in relation to the specific fluorescence of the intracellular NPe6 (fig. 1). During the treatment phase, SAFE 3000 examination also enabled the observation of complete photobleaching of the tumor, thereby assuring that no additional laser irradiation was needed during the procedure.

The sensitivity of SAFE 3000 for dysplastic lesion detection appears identical to the conventional fibered AF system. For instance, in both consecutive series published by Ikeda et al. [1], using fibered AFB (LIFE ${ }^{\circledR}$ system; first generation, XilliX, Richmond, $\mathrm{BC}$, Canada) or video-AF endoscopy, the sensitivity for the detection of moderate dysplasia or worse lesions were 67 and $87 \%$ for white light/ AF examination versus 65 and $90 \%$ for SAFE 3000, respectively [1].

The Olympus autofluorescence imaging bronchovideoscope system $\left(\mathrm{AFI}^{\circledR}\right)$ has a different design. It comprises three signals, including an excitation blue light (395$445 \mathrm{~nm}$ ) for AF and two different bands of reflected light: $\mathrm{G}(550 \mathrm{~nm})$ and $\mathrm{R}(610 \mathrm{~nm})$, which reflect red or blue signals [5]. In this fluorescence/reflectance system, precancerous lesions appear in magenta because they mix red/ blue reflected signals and lack the green fluorescence signal, while inflammation appears in blue because it contains a high concentration of hemoglobin that absorbs green and red light. In one study, the specificity of AFI for the detection of dysplasia or worse lesions appeared to be higher compared to the first generation of fibered AFB system (LIFE, Xillix), but the equivalence in terms of sensitivity was not clearly demonstrated (AFI sensitivity $=$ $80 \%$ vs. LIFE sensitivity $=98 \%$ ).

To date, none of the video-AF systems has been compared to the most recent versions of fiber-optic based AF systems that use dual light reflectance/fluorescence imaging such as D-Light ${ }^{\circledR}$ (Storz, Tuttlingen, Germany), 

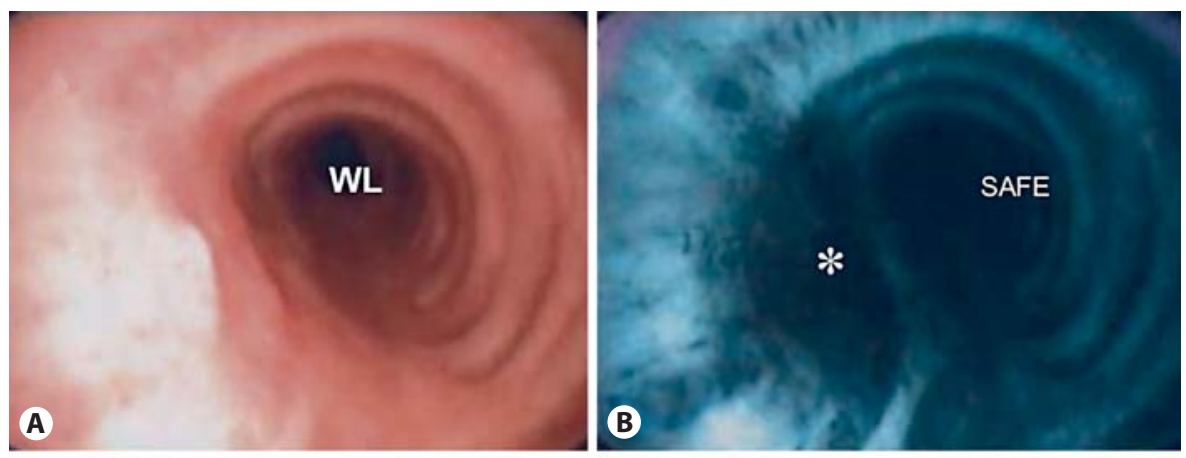

Fig. 1. Video-autofluorescence SAFE 3000 Imaging of an early lung cancer. A, B Dual white-light/autofluorescence imaging. The asterisk indicates the fluorescence defect of the superficial tumor. C, D Dual white-light/fluorescence imaging after intravenous administration of NPe6, a chlorine derivative. The asterisk indicates specific red fluorescence of the NPe6 incorporated into the tumor cells. Courtesy of Prof. Norihiko Ikeda, Tokyo Medical College, Japan.
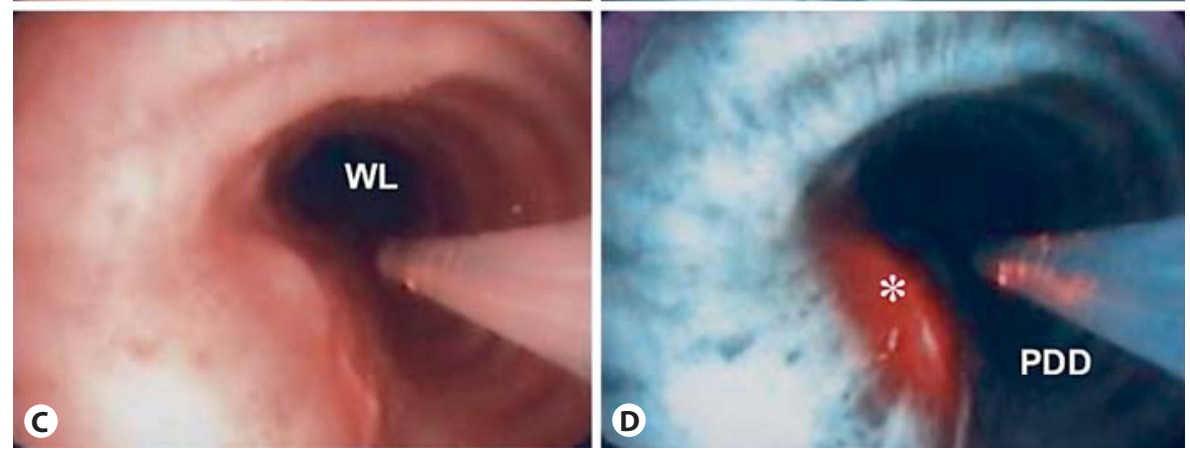

OncoLIFE $^{\circledR}$ (Novadaq, Richmond, BC, Canada) or Wolf (Richard Wolf Endoskope, Knittlingen, Germany) systems. However, close results seem likely to be found, except for the known better sensitivity of the white-light video compared to white-light fibered bronchoscopy.

To date, video-AF imaging systems remain significantly more expensive compared to AF fibered systems.

\section{Bronchial Microvascularization as a Target for Early}

Lung Cancer Detection: Narrow Band Imaging

Conventional video endoscopes and video cameras create images by superposing 3 monochromatic images for the red, green and blue channels. These 3 primary images are produced by either a 3 CCD microchip, or by the association of a monochrome CCD and a rotation disk with 3 RGB optical filters. The range of wavelengths in the conventional RGB broadband filters are: B 400-500 $\mathrm{nm}$; G 500-600 nm, and R 600-700 nm.

$\mathrm{NBI}$ is a new imaging modality that can be used to observe microvessel structure, using a narrow banding filter on an RGB sequential videoscope instead of the conventional RGB broadband filter [6]. The NBI filter includes three narrow windows at 400-430 nm (blue1), 420-470 $\mathrm{nm}$ (blue2), and 560-590 nm (green). The NBI-bluel filter covers the $415 \mathrm{~nm}$ maximum absorption peak of the hemoglobin. Therefore, in NBI mode, the vessels are specifically visualized as black images with high contrast.
NBI has been used to image the vascular pattern of esophageal and gastric mucosae. In conjunction with a high magnification video-gastroscope, NBI helps to the diagnosis of early cancers of the upper digestive tract without using topical contrast agents or chromoendoscopy [7].

Following the description of angiogenic bronchial dysplasia by the pathologists and its recognition by the endoscopists [8], Shibuya et al. [9] first made use of a high magnification broncho-videoscope in conjunction with fluorescence endoscopy to assess the microvasculature of the bronchial mucosae of high-risk patients in vivo. This made possible the description of areas of increased vessel growth and complex networks of tortuous vessels corresponding to the endoscopic appearance of angiogenic dysplasia, which enabled discrimination between areas of bronchitis and dysplasia. Subsequently, the same group made use of NBI in conjunction with high magnification video-bronchoscopy to describe a specific pattern of microvascular loops in angiogenic dysplasia and cancers that they termed 'dotted vessels' [6] (fig. 2).

NBI mode has recently been included by Olympus as a feature of their latest video-processor unit and made available along with the 180 video-bronchoscope series. Since then, a few reports have attempted to evaluate NBI modality with or without the use of AFB, or high-magnification bronchoscopy. A preliminary report by Herth et al. [10] using NBI along with Shibuya's description first 
Fig. 2. Specific vascular alteration imaging of lung cancer: narrow band imaging. A Dotted vessels. B Vascular loop. Courtesy of Prof. Felix Herth, Heidelberg University, Germany.
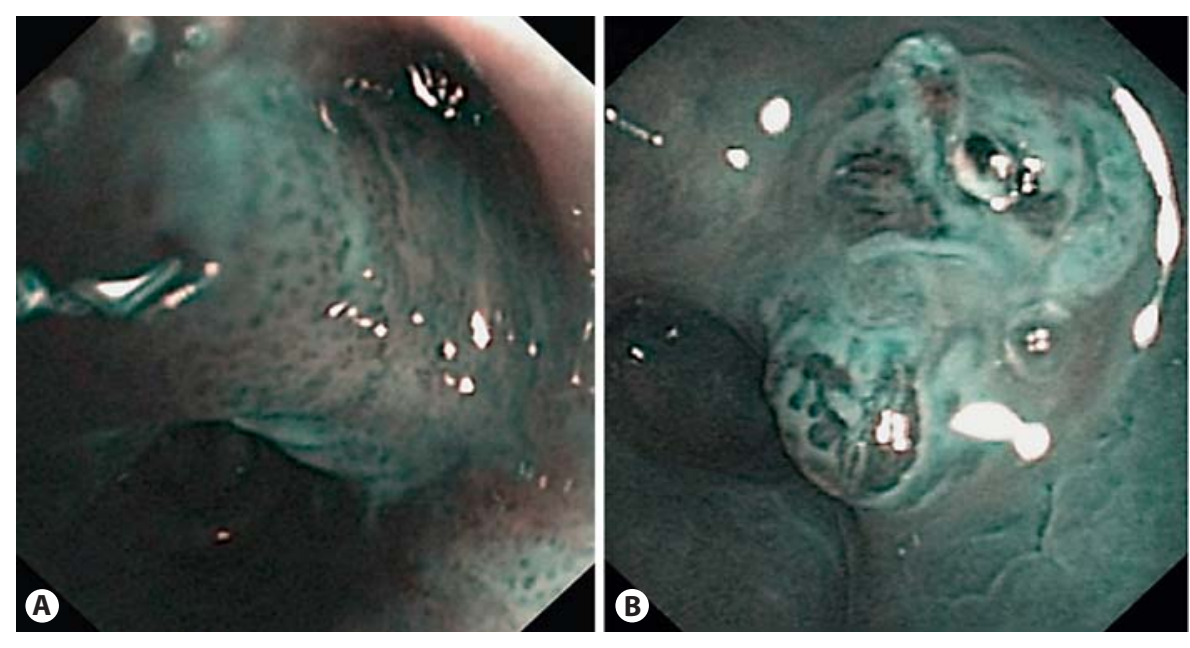

appeared encouraging, showing an increase in specificity for the diagnosis of precancerous lesions and a better discrimination between dysplasia and regular metaplasia compared to AF.

Two recent peer-reviewed publications better delineate the limits of this technique. A first study on a small number of patients (the majority with previously treated lung cancers) reported contrasting results, showing a small increase in sensitivity for the diagnosis of dysplasia or cancer, whereas no angiogenic dysplasia was found [11], with no advantage in invasive cancer.

Recently, Herth et al. [12] published work on the combined use of AFI together with NBI and compared the results of both modalities to white-light endoscopy. The study was conducted in high-risk patients without clinical or radiological evidence of lung cancer. White-light endoscopy was performed first, followed by either NBI then AFI or AFI then NBI in a random order. Data on 62 patients and 103 separate biopsy sites were analyzed. Five roentgenographically occult invasive lung cancers were diagnosed, which were detected by all three modalities. Eighteen high-grade lesions (moderate dysplasia to CIS) on 17 patients were diagnosed during the study. This provided a relative sensitivity compared to white light of 3.7 for AFI and 3.0 for NBI, with better specificity for NBI.

These data indicate that NBI could be useful without magnifying bronchoscopes, in contrast to what is usually agreed upon for the upper gastrointestinal tract [13]. Further study on bronchial NBI should focus on high-risk patients with or without angiogenic dysplasia, using precise descriptors of bronchial microvasculature.

Finally, a close endoscopic imaging modality called FICE ${ }^{\circledR}$ has been made available by Fuginon (Tokyo, Ja- pan). FICE uses electronic filters with a large variety of combinations to enhance mucosal vascular contrast. This modality has not been evaluated in bronchial exploration so far.

\section{Towards Microscopic Histology Level Imaging: Optical Coherence Tomography}

OCT is an optical imaging method that can offer microscopic resolution for visualizing structures at and below a tissue surface [14].

The principle of OCT is similar to ultrasound imaging, but uses near infrared light instead of sound waves. Using ultrasound, the imaging is accomplished by measuring the delay time (echo delay) for an incident ultrasonic pulse to be reflected back from structures within tissue. Because the velocity of sound is relatively slow, this delay time can be measured electronically. However, since the velocity of light is 200,000 times that of sound, measurements of light delay cannot be performed directly by electronic techniques. Therefore, a technique known as low coherence interferometry is used.

By scanning the optical beam across the sample, tomographic images are produced in a manner similar to radar, and represent a cross-sectional image of the optical reflectance properties within tissue. Unlike ultrasound, light waves do not require liquid-based coupling medium and thus are more compatible with airway imaging.

The resolution of OCT, in both the axial and the lateral dimensions, is more than an order of magnitude higher than that of ultrasonic examination, potentially enabling optical biopsy. The currently commercialized 
OCT device for lung exploration (collaboration of LightLab imaging, Boston, Mass., USA, and Pentax, Tokyo, Japan) can produce images of the respiratory tract with an axial resolution of $15-20 \mu \mathrm{m}$ and a lateral resolution of 20-30 $\mu \mathrm{m}$ for a depth of focus of 1-2 $\mathrm{mm}$. The system uses a small optical probe with an outer diameter of $1.5 \mathrm{~mm}$ that is inserted into the bronchoscope's working channel and allows radial scanning of the airways circumferentially to generate transluminal OCT images in real time.

In the pioneer work by Tsuboi et al. [15], OCT was used on 7 resected lung samples and in 5 patients during bronchoscopy to collect images from normal bronchus and tumors. Clear imaging was seen to a depth of $2 \mathrm{~mm}$ to the cartilage layer. This study clearly visualized the layer between the epithelium and basement membrane and demonstrated that early invasive cancers can be distinguished from normal bronchial epithelium and pre-invasive cancers based on OCT images.

Ex vivo OCT images of the bronchial epithelial structures were also published by Whiteman et al. [14] who clearly visualized differences between healthy airway and the presence of inflammation or tumor. Ex vivo crosssectional OCT images precisely delineated the following anatomic bronchial components: epithelium, lamina propria, smooth muscle, mucus glands and cartilage.

Recently, these findings have been further improved by studying OCT in conjunction with fluorescence bronchoscopy in 138 volunteer heavy smokers and 10 patients with lung cancer in vivo [16]. A total of 281 OCT images and the corresponding bronchial biopsies were obtained. The histopathology of these areas included 145 normal/ hyperplasia, 61 metaplasia, 39 mild dysplasia, 10 moderate dysplasia, 6 severe dysplasia, 7 CIS, and 13 invasive carcinomas. Normal epithelium or hyperplasia appeared as one or two cell layers above a highly scattering basement membrane and upper submucosa. A progressive increase in the epithelial thickness was found to parallel the severity of the histopathology grade. Epithelial thickness was also significantly different between invasive cancer and CIS. In addition, cell nuclei became more readily visible in high-grade dysplasia or CIS. The basement membrane was still intact in CIS but became discontinuous or no longer visible with invasive cancer.

Finally, OCT has been used to measure airway wall dimensions, a finding that may have important implications in small airway monitoring in COPD and other non-neoplastic diseases [17].

These data indicate that OCT may replace endobronchial ultrasound for the assessment of early cancer invasion into the bronchial wall, whereas the capabilities to image cartilage invasion is not clear from the published in vivo data.

While the technique appears to have great potential for management of early lung cancer/precancerous conditions, current OCT images are far from the pathological grade of optical biopsies, which indicates that the technique cannot yet replace biopsy sampling for precancerous/early cancer diagnostics or monitoring studies.

\section{Towards in vivo Imaging of the Respiratory Cells: Confocal Microendoscopy}

The principle by which confocal microscopy images a thin slide of a sample relies on both the use of a narrow point source on the illumination path and of a small aperture or pinhole on the light detection path. This results in the rejection of out-of-focus information from the material above and below a very thin plane of focus.

The currently available microendoscopic devices use the principle of probe-based confocal fluorescent microscopy, i.e. fluorescent imaging of a thin slice within a tissue, where the objective is replaced by an optical fiber. Such systems have recently been applied to the explorations of several organs, including the gastrointestinal tract, and more recently to the proximal and distal airways in vivo $[18,19]$.

Respiratory fluorescence microendoscopes use a flexible miniprobe with an outer diameter of $1 \mathrm{~mm}$ that is introduced into the working channel of the bronchoscope and applied to the tissue. The miniprobe is made of thousands of fiber cores which are scanned, one at a time, by a laser source in a raster fashion, by the use of two rapidly moving mirrors. This bundle conducts the light back and forth from the imaged area at the tip of the miniprobe. According to this design, each fiber core is in essence its own pinhole, thereby assuring the confocal properties of the system. This system is also described as 'fibred confocal fluorescent microscopy' (FCFM). One device series called Cell-viZio ${ }^{\circledR}$ Lung (Mauna Kea Technologies, Paris, France) is currently commercialized. Two different wave lengths are available. The Cell-viZio $488 \mathrm{~nm}$ is used for AF microimaging of the respiratory tract. Another device, at $660 \mathrm{~nm}$ excitation, can be used for epithelial cell imaging after topical application of exogenous fluorophores such as methylene blue (MB) [20].

The Cell-viZio devices have a lateral resolution of $3 \mu \mathrm{m}$, a field of view of $600 \mu \mathrm{m}$, a depth of focus of $0-50$ $\mu \mathrm{m}$ and produce real-time imaging at 9-12 frames/s. For 
Fig. 3. Optical coherence tomography (OCT) of occult lung cancer during bronchoscopy. White arrows: superficial tumor. A, C White light bronchoscopy imaging. B, D Corresponding OCT in vivo imaging. A, B Correspond to the superficial tumor imaged in figure 1 . Courtesy Prof. Norihiko Ikeda, Tokyo Medical College, Japan.

Fig. 4. Confocal microendoscopy of the human proximal airways in vivo. A Confocal imaging of the basement membrane, autofluorescence at $488 \mathrm{~nm}$ illumination (Cell-viZio Lung-488). B In vivo imaging of the normal epithelial layer after topical application of $0.1 \%$ methylene blue, and $660 \mathrm{~nm}$ illumination (Cell-viZio 660). C Microimaging of methylene blue reabsorption by the bronchial lymphatics. (Cell-viZio 660). D In vivo confocal microendoscopy of a large cell carcinoma of the bronchus, same patient as in B (Cell-viZio 660). All images: Prof. L. Thiberville, Rouen University Hospital, Rouen, France.
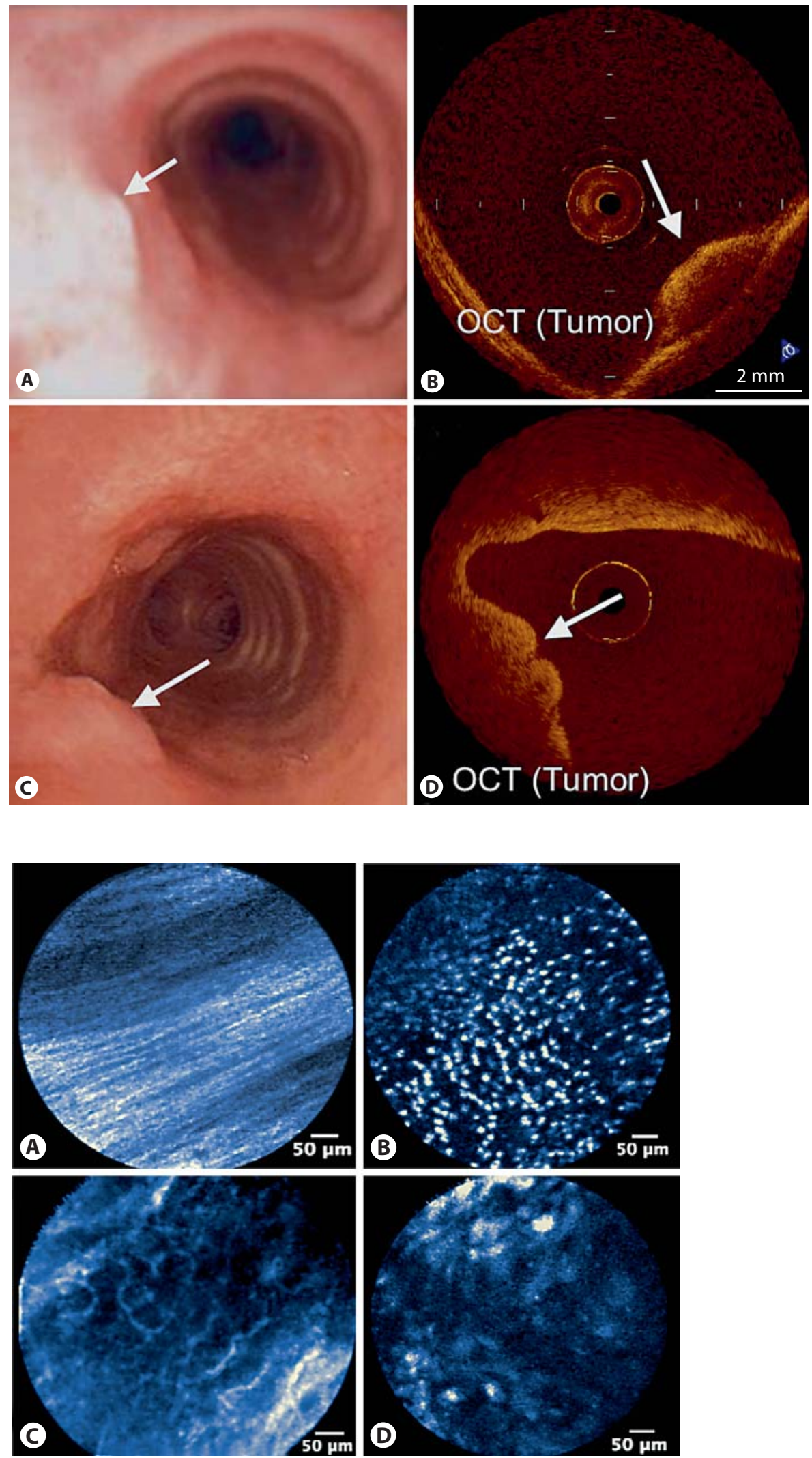

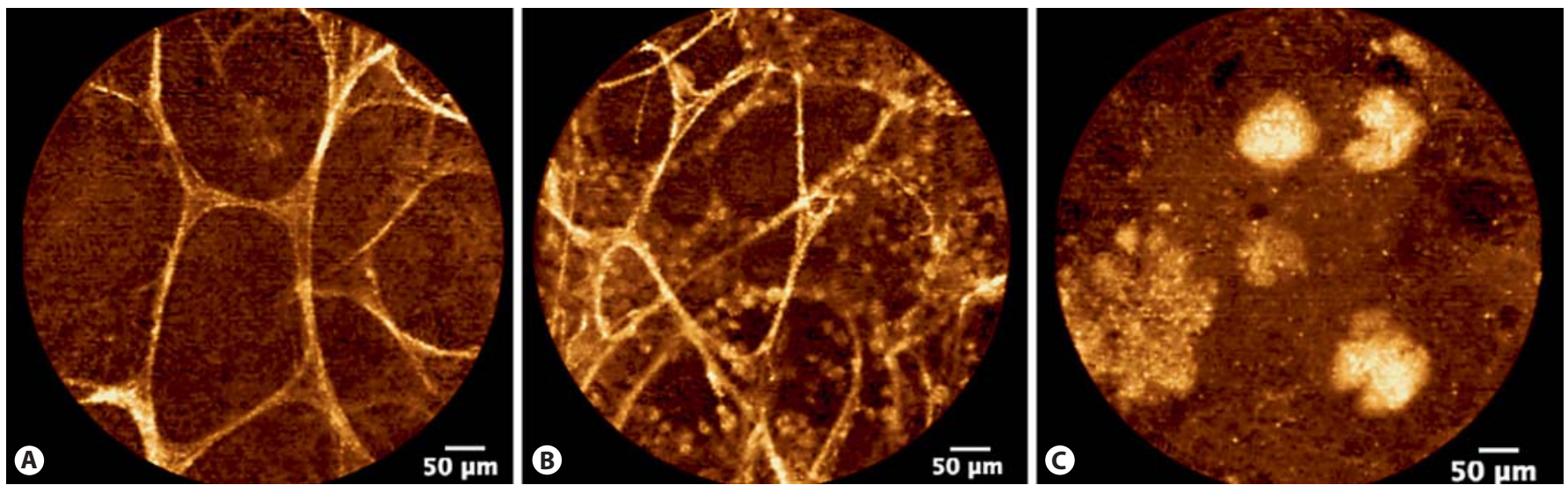

Fig. 5. Confocal fluorescence microendoscopy of the distal lung (autofluorescence, $488 \mathrm{~nm}$ excitation, Cell-viZio Lung-488). A Normal axial elastic backbone of an alveolar duct. Nonsmoking subject. B Normal alveoli showing cellular infiltration by fluorescent cells, presumably alveolar macrophages, in an actively smoking patient. C Confocal fluorescence microimag- ing in a case of pulmonary alveolar proteinosis, showing characteristic large fluorescent globular structures into the distal airways of an active smoker. Cell-viZio Lung-488 imaging. All images: Prof. L. Thiberville, Rouen University Hospital, Rouen, France. in vivo imaging, the miniprobe is applied onto the bronchial wall surface, or can be advanced into a distal bronchiole in order to image the most distal acinar parts of the lung down to the subpleural regions $[18,19]$.

In non-smokers, the 488 -nm excitation device images the AF of the elastin that is contained in the basement membrane of the proximal airways and that participates in the axial backbone of the peripheral interstitial respiratory system $[18,19]$. In smokers, a specific tobacco tarinduced fluorescence also allows in vivo macrophage and alveolar wall imaging [18].

Due to the fluorescent properties of the respiratory tissue, and the depth of focus of the system $(50 \mu \mathrm{m}$ below the contact surface), the device images the AF arising from the first layers of the bronchial epithelial and subepithelial connective tissue. At $488 \mathrm{~nm}$ excitation, FCFM produces very precise microscopic fluorescent images of the bronchial basement membrane zone in real time, revealing a mat of fibers mainly oriented along the longitudinal axis of the airways, as well as small openings (100$200 \mu \mathrm{m}$ wide) corresponding to the origins of the bronchial glands [19]. However, flavin cellular AF of normal tissue appears too weak to allow $488 \mathrm{~nm}$ Cell-viZio imaging of the epithelial layer without addition of exogenous fluorophore.

Previous studies have shown that endoscopic FCFM could be used to study specific basement membrane remodeling alterations in benign or malignant/premalignant bronchial alterations. The FCFM microstructure of the bronchial walls underlying premalignant epithelia is significantly altered. In these precancerous conditions, the elastic fibred pattern of the lamina reticularis is absent or disorganized in almost every pre-invasive lesion, supporting the hypothesis of an early degradation of the basement membrane components in pre-invasive bronchial lesions [19]. However, in its AF mode, the absence of epithelial cell visualization does not allow the technique to clearly separate the different grades of progression of the precancerous bronchial lesions, such as metaplasia/ dysplasia/CIS, from each other.

In order to be successfully applied to the exploration of precancerous/cancerous bronchial epithelium, the FCFM technique needs to be coupled with the use of a non-toxic cellular dye, acting as an exogenous fluorophore. Ex vivo studies have shown that the resolution of the system is not a limitation to nuclear or cellular imaging [21]. Exogenous fluorophores that could be activated at $488 \mathrm{~nm}$ have not yet been used in this application.

$\mathrm{MB}$ is a non-toxic agent which is commonly used during bronchoscopy for the diagnosis of bronchopleural fistulae. MB is also used in gastroenterology for chromoendoscopic detection of precancerous lesions, as well as for in vivo microscopic examination of the gastrointestinal tract and bronchus using a novel endocytoscopic system [22]. MB is also a potent fluorophore which enters the nuclei and reversibly binds to the DNA, before being reabsorbed by the lymphatics. In order to give a fluorescent 
signal, MB needs to be excited around $660 \mathrm{~nm}$, and is therefore accessible to FCFM intravital imaging using this excitation wave length.

Preliminary study has demonstrated that Cell-viZio 660/topical MB makes it possible to reproducibly image the normal and cancerous epithelial layer of the main bronchi [20] as well as cellular patterns of solid peripheral lung nodules $[23,24]$ (fig. 4). Future studies using this technique could make it possible to differentiate normal, premalignant and malignant alterations at the microscopic level but, in contrast to OCT, may not differentiate between CIS and early invasive cancers. If this strategy is successful, FCFM may become a very powerful technique for in vivo diagnosis of early malignant and premalignant conditions of the bronchial tree, allowing analysis of both the epithelial and subepithelial layers during the same procedure.

Another advantage of confocal microendoscopy using the miniprobe approach is that the technique makes it possible to image distal structures in vivo, such as the alveolar ducts, sacs and intra-acinar, extra-alveolar and capillary microvessels. A specific tobacco-induced fluorescence is observed in smoking subjects (fig. 5).
The potential applications for in vivo distal lung imaging using miniprobe-based confocal endoscopy appear to be wide. Some limitations of the technique could be predicted from its basic principles, such as artifacts linked to compression of fragile parenchymal lung structures, as well as difficulties in interpretation of an imaging technique mainly based on elastin network assessment. However, preliminary results are encouraging in specific diffuse or focal lung diseases, such as in pulmonary alveolar proteinosis [25], diffuse emphysema [26], or peripheral lung nodules [23].

In conclusion, emerging endoscopic approaches now provide new insight into bronchology, in extending the field of exploration from the proximal bronchus down to the most distal part of the lungs, and from the macroscopy to in vivo cellular imaging. In the near future, these exciting technologies may enable in vivo, minimally invasive, 'pathological grade' evaluation of abnormal bronchial or parenchymal lung tissue. Whereas promising pioneer work has recently been published, they will require careful assessment before their place in the evaluation strategy of early lung cancer and other lung diseases can be delineated.

\section{References}

$>1$ Ikeda N, Hayashi A, Iwasaki K, Honda H, Tsuboi M, Usuda J, Kato H: Comprehensive diagnostic bronchoscopy of central type early stage lung cancer. Lung Cancer 2007;56: 295-302.

$\checkmark 2$ Ikeda N, Honda H, Hayashi A, Usuda J, Kato Y, Tsuboi M, Ohira T, Hirano T, Kato H, Serizawa $\mathrm{H}$, et al: Early detection of bronchial lesions using newly developed videoendoscopy-based autofluorescence bronchoscopy. Lung Cancer 2006;52:21-27.

$\checkmark 3$ Lee P, Brokx HA, Postmus PE, Sutedja TG: Dual digital video-autofluorescence imaging for detection of pre-neoplastic lesions. Lung Cancer 2007;58:44-49.

$\checkmark 4$ Usuda J, Tsutsui H, Honda H, Ichinose S, Ishizumi $\mathrm{T}$, Hirata $\mathrm{T}$, Inoue $\mathrm{T}$, Ohtani $\mathrm{K}$, Maehara S, Imai $\mathrm{K}$, et al: Photodynamic therapy for lung cancers based on novel photodynamic diagnosis using talaporfin sodium (NPe6) and autofluorescence bronchoscopy. Lung Cancer 2007;58:317-323.

$\checkmark 5$ Chiyo M, Shibuya K, Hoshino H, Yasufuku K, Sekine Y, Iizasa T, Hiroshima K, Fujisawa $\mathrm{T}$ : Effective detection of bronchial preinvasive lesions by a new autofluorescence imaging bronchovideoscope system. Lung Cancer 2005;48:307-313.
-6 Shibuya K, Hoshino H, Chiyo M, Iyoda A Yoshida S, Sekine Y, Iizasa T, Saitoh Y, Baba M, Hiroshima $K$, et al: High magnification bronchovideoscopy combined with narrow band imaging could detect capillary loops of angiogenic squamous dysplasia in heavy smokers at high risk for lung cancer. Thorax 2003;58:989-995.

7 Gheorghe C: Narrow-band imaging endoscopy for diagnosis of malignant and premalignant gastrointestinal lesions. J Gastrointestin Liver Dis 2006;15:77-82.

$\checkmark 8$ Keith RL, Miller YE, Gemmill RM, Drabkin HA, Dempsey EC, Kennedy TC, Prindiville S, Franklin WA: Angiogenic squamous dysplasia in bronchi of individuals at high risk for lung cancer. Clin Cancer Res 2000;6: 1616-1625

-9 Shibuya K, Hoshino H, Chiyo M, Yasufuku K, Iizasa T, Saitoh Y, Baba M, Hiroshima K, Ohwada H, Fujisawa T: Subepithelial vascular patterns in bronchial dysplasias using a high magnification bronchovideoscope. Thorax 2002;57:902-907.

10 Herth FJ, Eberhardt R, Ernst A: Narrow band imaging (NBI) for early lung cancer detection. Chest 2006;130:146S-a (meeting abstract).
11 Vincent BD, Fraig M, Silvestri GA: A pilot study of narrow-band imaging compared to white light bronchoscopy for evaluation of normal airways and premalignant and malignant airways disease. Chest 2007;131: 1794-1799.

12 Herth FJ, Eberhardt R, Anantham D, Gompelmann D, Zakaria MW, Ernst A: Narrow-band imaging bronchoscopy increases the specificity of bronchoscopic early lung cancer detection. J Thorac Oncol 2009;4: 1060-1065

-13 Tahara T, Shibata T, Nakamura M, Yoshioka D, Okubo M, Arisawa T, Hirata I: Gastric mucosal pattern by using magnifying narrow-band imaging endoscopy clearly distinguishes histological and serological severity of chronic gastritis. Gastrointest Endosc 2009;70:246-253.

14 Whiteman SC, Yang Y, Gey van Pittius D, Stephens M, Parmer J, Spiteri MA: Optical coherence tomography: real-time imaging of bronchial airways microstructure and detection of inflammatory/neoplastic morphologic changes. Clin Cancer Res 2006;12:813818.

15 Tsuboi M, Hayashi A, Ikeda N, Honda H, Kato Y, Ichinose S, Kato H: Optical coherence tomography in the diagnosis of bronchial lesions. Lung Cancer 2005;49:387-394. 
-16 Lam S, Standish B, Baldwin C, McWilliams A, leRiche J, Gazdar A, Vitkin AI, Yang V, Ikeda N, MacAulay C: In vivo optical coherence tomography imaging of preinvasive bronchial lesions. Clin Cancer Res 2008; 14 : 2006-2011.

-17 Coxson HO, Quiney B, Sin DD, Xing L, McWilliams AM, Mayo JR, Lam S: Airway wall thickness assessed using computed tomography and optical coherence tomography. Am J Respir Crit Care Med 2008;177:12011206.

-18 Thiberville L, Salaün M, Lachkar S, Dominique S, Moreno-Swirc S, Vever-Bizet C, Bourg-Heckly G: Human in vivo fluorescence microimaging of the alveolar ducts and sacs during bronchoscopy. Eur Respir J 2009;33:974-985

$>19$ Thiberville L, Moreno-Swirc S, Vercauteren T, Peltier E, Cave C, Bourg Heckly G: In vivo imaging of the bronchial wall microstructure using fibered confocal fluorescence microscopy. Am J Respir Crit Care Med 2007; 175:22-31.
20 Thiberville L, Salaün M, Moreno-Swirc S, Bourg Heckly G: In vivo endoscopic microimaging of the bronchial epithelial layer using $660 \mathrm{~nm}$ fibered confocal fluorescence microscopy and topical methylene blue. Eur Respir J 2007;30(suppl 51):712s (meeting abstract).

21 Laemmel E, Genet M, Le Goualher G, Perchant A, Le Gargasson JF, Vicaut E: Fibered confocal fluorescence microscopy (CellviZio) facilitates extended imaging in the field of microcirculation. A comparison with intravital microscopy. J Vasc Res 2004;41: 400-411.

22 Shibuya K, Yasufuku K, Chiyo M, Nakajima T, Fujiwara T, Nagato K, Suzuki H, Iyoda A, et al: Endo-cytoscopy system is a novel endoscopic technology to visualize microscopic imaging of the tracheobronchial tree. Eur Respir J 2008;32(suppl 52):263s (meeting abstract).
23 Thiberville L, Salaün M, Lachkar S, MorenoSwirc S, Bourg-Heckly G: In vivo confocal endomicroscopy of peripheral lung nodules using 488/660 $\mathrm{nm}$ induced fluorescence and topical methylene blue. Eur Respir J 2008; 32(suppl 52):263s (meeting abstract).

24 Thiberville L, Salaün M, Lachkar S, Dominique S, Moreno-Swirc S, Vever-Bizet C, Bourg Heckly G: In vivo confocal fluorescence endomicroscopy of lung cancer. J Thorac Oncol 2009;4:S49-S51.

25 Salaün M, Roussel F, Hauss PA, Lachkar S, Thiberville L: In vivo imaging of pulmonary alveolar proteinosis using confocal endomicroscopy. Eur Respir J 2010, in press.

26 Thiberville L, Salaün M, Hauss PA, Lachkar $S$, Dominique S: In vivo microimaging of the alveolar capillary network during alveoscopy. Eur Respir J 2009;33(suppl 53):91s (meeting abstract). 